\title{
Vulnerabilidad, dignidad y justicia: Valores éticos fundamentales en un mundo globalizado*
}

\begin{abstract}
Stå Isett, Sturla J .***
* Trabajo presentado en el Encuentro internacional: “Las dimensiones éticas del desarrollo: Los Nuevos Desafíos Éticos del Estado, la Empresa y la Sociedad Civil". Belo Horizonte, Brasil, 3 y 4 de Julio de 2003. Agradezco la invitación de formar parte en la junta de asesores de iniciativa 'Etica y Desarrollo del BID', y de contribuir en esta gran conferencia sobre los nuevos desafíos éticos del estado, la empresa y la sociedad civil. ** Dr. en Teología. Profesor asociado y director del programa de investigación: Religión in a globalised age [RIGA] Facultad de Teología Universidad de Oslo, Noruegas. E-mail:
\end{abstract}

\section{j.stalsett@teologi.uio.no}

\section{Resumen}

En el ensayo se reflexiona sobre un aspecto ético vinculado con la dignidad y la justicia en el contexto de los procesos de globalización que afectan desigualmente a toda la humanidad: la vulnerabilidad. Se propone que ella sea asumida como un valor antropológico y ético; esto conlleva a construirla histórico-conceptualmente como Vulnerabilidad Compartida, por cuanto representa un problema que actualmente impacta a todos los habitantes de la tierra, aún cuando los grados de afectación se encuentren asimétricamente distribuidos.

Palabras clave: Vulnerabilidad, justicia, globalización, dignidad, ética.

\begin{abstract}
In this essay, an ethical aspect related to dignity and justice in the context of the globalization processes which unequally affect all of humanity, is analyzed: the aspect of vulnerability. It is proposed that vulnerability be accepted as an anthropological and ethical value; which leads to the historical-conceptual construction of Shared Vulnerability, since it represents a problen that presently is affecting all the inhabitants of the earth, even when the degree of affectation is asymetrically distributed.
\end{abstract}

\section{Vulnerability, Dignity and J ustice: Fundamental Ethical Values in a Globalized World}


Key words: Vulnerability, justice, globalization, dignity, ethics.

Recibido: 03-12-19. Aceptado: 04-01-19

\section{I ntroducción: Dos dimensiones de 'lo ético'}

Existe hoy un interés fuerte y renovado en cuestiones éticas. Los desafíos, dilemas, deficiencias e injusticias del mundo globalizado nos rodean por todas partes. El programa 'Etica y Desarrollo' del Banco Interamericano de Desarrollo es una muestra de tal interés, al mismo tiempo que lo promueva y profundiza. Entiendo que la respuesta a este programa ha sido impresionante, lo cual muestra la necesidad y relevancia de poner el tema ético en la agenda ligada a las grandes transformaciones latinoamericanas y globales.

El tema sobre el que aquí discuto se puede presentar en una forma abreviada como la ética y la globalización, sobre el cual existe una voluminosa producción dentro de la que destacan, entre otros, los trabajos de Singer (2002) y Sáenz (2002). Aunque lo voy a desarrollar en un nivel bastante generalizado, espero que mis aportes puedan funcionar como plataforma o marco de referencia ética al tratamiento de los desafíos más concretos a los que hay que darle respuesta tanto en el ámbito político como económico.

Ahora, obviamente hay varias concepciones y sentidos de 'lo ético'. Para nuestros propósitos aquí, creo que sería útil distinguir entre ética como la búsqueda de la actitud correcta, la buena conducta del ser humano por un lado, y ética como la buena vida o la buena sociedad por el otro lado. Dicho de manera simple, la diferencia se trata de si se pone el mayor énfasis en cómo se camina, o en hacia dónde se va. En la primer concepción de la ética hay un énfasis en la calidad ética/moral de los procedimientos. Se enfoca si la manera en que se realiza esta o tal operación o actividad es 'buena', es decir, si se la lleva a cabo con rectitud, buenas intenciones, honestidad, transparencia, entre otras. Esta es ética de profesión, muchas veces con un fuerte carácter individualista. Sin duda, esta dimensión de la ética tiene gran relevancia en América Latina y el resto del mundo hoy. Los temas de corrupción, crisis de confianza, mal uso de poder, malversación de fondos públicos, favoritismos, impunidad, entre otros, son vigentes y reclaman tratamiento, discusión y confrontación. En el mundo de hoy se necesitan personas, mujeres y hombres, de gran estatura moral en quienes se puede confiar. Gente que siguen las reglas del juego.

Sin embargo, este no es suficiente. Porque poco ayuda que se camine correctamente 
hacia una meta errónea; no sirve para nada seguir las reglas, si estas en si son malas. Es fundamental por lo tanto llegar a la otra, más profunda dimensión de lo ético. Esta comprende la calidad ética fundamental de las metas, y la sustancia y legitimidad de las reglas. La pregunta será: ¿Qué es la buena vida para el ser humano? ¿Qué es, en fin, la buena sociedad? Esta concepción de la ética como búsqueda de la buena vida puede parecer superficial. Pero no es la misma pregunta que se nos responde tan simplísticamente en los comerciales. Esta pregunta por la vida buena en un sentido éticomoral más bien tiene raíces profundas en la tradición aristotélica, tanto como la tradición hebréa-cristiana. Es importante añadir en nuestro tiempo algo que le da un acento crítico explícito: ¿Qué es la buena vida para todos y todas en comunidad, y en particular, para aquellas personas que por algunas razones han sido excluidas? De ahí la pregunta por el bien común y el derecho del marginalizado, que no tiene por qué estar en contradicción con el anhelo y derecho individual de vivir en plenitud y dignidad.

Lo que se propone se encuentra en correspondencia con esta segunda dimensión ética. Tratando de acercar la problemática de la buena vida y buena sociedad desde la perspectiva de los valores que las caracterizan, quiero proponer tres valores éticos fundamentales en el mundo globalizado. Estos valores son la vulnerabilidad, la dignidad y la justicia. Ahora, puede sorprender que propongo la vulnerabilidad como un valor ético. Por eso empiezo con este. Y además, siendo un concepto no sólo fundamental sino también ambiguo o dialéctico, me parece fructífero utilizarlo como punto de partida al tratar de definir lo que se quiere decir con 'un mundo globalizado'.

\section{Globalización: Vulnerabilidad compartida, pero asim étrica}

¿Qué entendemos aquí por ‘globa- lización'? Entre la gran multitud de definiciones que hoy se presentan, quisiera proponer una perspectiva parcial, pero-creo-iluminadora y útil para la reflexión ética (Held and McGrew, 2000). Los orígenes históricos de la globalización los encontramos en la conciencia de la posibilidad del acceso a todo el globo - el reconocimiento de la tierra como un todo que es accesible y a disposición para (algunos de) los seres humanos. Obviamente, el año 1492 es sumamente significativo en este sentido (Dussel, 1998; Hinkelammert, 1999), así como también el descubrimiento del globo redondo y la revolución industrial con su división de trabajo y explotación global. Sin embargo, globalización en sentido propio sobre todo se refiere a la situación actual en el cual esta disponibilidad o accesibilidad al todo corresponde a las compañías transnacionales, el capitalismo global y el mercado ilimitado.

Ahora, propongo que la globalizaci ón en el sentido crítico se refiere al lado negativo o los 
límites de esa accesibilidad y disponibilidad global que, aunque violentamente presente ya en el proceso de la conquista del siglo $\mathrm{XV}$, se presentan en manera más sistemática desde el siglo pasado. Esto es la conciencia de que el globo como un todo es vulnerable, y de que esta vulnerabilidad es compartida entre todo los habitantes de la tierra. Geopolítica- y militarmente esto se expresa en la sobre-capacidad de destrucción mutua y total del balance del terror en la edad nuclear. Ecológicamente esta vulnerabilidad global se muestra por primera vez en el informe del Club de Roma Limits to Growth de 1972, cuando se advierte que el crecimiento tiene límites - que se acerca el momento en que el globo ya no aguanta más contaminación humana, y luego en el Informe de la Comisión Brundtland (Our Common Future, 1987), el Protocolo de Kyoto de 1997 y las Conferencias en Río de Janeiro, 1992 y Johannesburgo, 2002 sobre el ambiente y el desarrollo. En las últimas décadas el sentido de una vulnerabilidad global y mutua ha crecido a través de las crisis financieras de México, Asia y Argentina, y con epidemias como el VIH-SIDA y, ahora, el SARS. Por último, los ataques terroristas del 11 de septiembre 2001 y su respuesta en la guerra contra el terrorismo representan la culminación de este proceso de globalización como sentido de vulnerabilidad global, compartida.

Es importante notar que aunque esta vulnerabilidad es compartida, en el sentido de que nadie en principio la puede escapar, a la vez es claramente asimétrica. No afecta a todos por igual, de igual manera o al mismo grado. Al contrario, un resultado innegable de la globalización es la polarización económica y exclusión social (Bauman, 1998). En la globalización hay 'muchos perdedores y pocos ganadores', como bien ha demostrado el Dr. Bernardo Kliskberg (2003a), coordinador de la iniciativa de Ética y Desarrollo en el BID y organizador de este evento. “El mundo tiende a dividirse cada vez más entre ganadores, y perdedores. Estos últimos superan muchas veces a los primeros. Sobre 6500 millones de personas, 3000 millones gana menos de dos dólares diarios, y otros 1500 millones, menos de un dólar diario. Son pobres. Su número creció en relación a 1980. Las distancias sociales aumentan. Las diferencias de ingresos entre el $20 \%$ de la población que vive en los países más ricos y el $20 \%$ que vive en los más pobres era de 30 a 1 en 1960, pasó a ser de 60 a 1 en 1990, y en 1997 ya había llegado a 74 a 1" (Kliskberg, 2003b).

Si la globalización representa 'un mundo de oportunidades', estas de hecho son oportunidades exclusivas para una élite mundial. Recientemente el Gobierno de Noruega presentó un Libro Rojo sobre la globalización, Ilamado “Un mundo de oportunidades. La edad de la globalización y sus desafíos" (En verden av muligheter - globaliseringens tidsalder og dens utfordringer 2003), en el cual, en mi opinión, se presenta una visión demasiada armónica de la globalización. 
Paradójicamente parece ser un aspecto constitutivo del proceso de globalización actual que en el mismo movimiento en que acaparan el todo, las fuerzas globalizadoras echan cada vez mas gente para afuera. Hay un mundo creciente 'afuera', un mundo que sobra, un mundo que para el sistema parece ser desechable. Globalización también es el 'crecimiento paradójico de un mundo afuera del globo' (Sáenz, 2002:3). Es esta marginación y exclusión, que aquí llamo la asimetría de la vulnerabilidad compartida, que nos lleva a las cuestiones de poder y de justicia - cuestiones fundamentalmente éticas. Dicho de otro modo, al hablar de la ética frente a la globalización me refiero a la conciencia sobre esta vulnerabilidad compartida pero asimétrica, y la voluntad de tomar responsabilidad y actuar frente a esta asimetría destructiva. El momento ético es cuando se reconoce esta vulnerabilidad asimétrica, y se la asume como tarea personal y colectiva. Ética es vulnerabilidad reconocida y asumida.

\section{Vulnerabilidad como valor antropológico y ético}

La globalización es en este sentido una crisis. Muchos lo ven, como p.ej. Franz Hinkelammert, como una crisis de la civilización:

Esta crisis de la civilización se refiere a toda la modernidad surgida desde el siglo XV y construida sobre la eficiencia en términos de relaciones medio-fin aisladas y parcializadas. Hoy tenemos que supeditar esta eficiencia a otra, que es la eficiencia de la sostenibilidad de la vida humana en una naturaleza sostenible (Hinkelammert, 1999: 15).

La palabra 'crisis' quiere decir juicio y oportunidad de cambio. ¿Cuáles son los conceptos o valores éticos que nos muestran por dónde hay que buscar las soluciones a esta crisis mundial? ¿Cuáles son las calidades que caracterizarán una globalización alternativa, una sociedad global realmente humana?

Aunque parezca paradójico, quiero continuar aquí con el concepto de vulnerabilidad. Normalmente, por vulnerabilidad se entiende una debilidad, una fragilidad. Con buena razón. Vulnerabilidad significa capacidad de ser herido. Nadie quiere ser herido. Por lo tanto, el otro lado de la vulnerabilidad es derecho a la protección y necesidad de seguridad en sentido amplio.

Pero eso no es todo. Cuando se entiende exclusivamente así, la búsqueda de la eliminación de la vulnerabilidad humana lleva a una preocupación excesiva por seguridad, que pueda tener consecuencias deshumanizantes. Esto es porque vulnerabilidad en el 
sentido profundo es una característica humana indeleble y constituyente. Ser humano es ser vulnerable. Un ser invulnerable sería un ser inhumano. Sin vulnerabilidad humana, ningún ser es humano. Vulnerabilidad es una condición antropológica fundamental, y por lo tanto imborrable. Este presupuesto antropológico tiene consecuencias éticas. Porque la vulnerabilidad humana también significa apertura hacia el Otro (Lévinas, 1972: 102-106).

Es el fundamento de sensibilidad, compasión y comunidad. Sin vulnerabilidad humana, o más bien, sin reconocimiento de la vulnerabilidad propia, no habría condiciones para reconocer la vulnerabilidad del otro y la demanda ética que presenta. Sin vulnerabilidad, ningún reconocimiento del desafío ético (Løgstrup, 1989).

Es por eso que el sueño de invulnerabilidad, tan central en el proyecto moderno, tan querido por los Imperios históricos y actuales, es en fin un sueño inmoral. Es inmoral por ser deshumanizante. La preocupación excesiva de seguridad está paradigmáticamente expresada en construcciones de muros: el muro de Berlín, el nuevo muro que los Israelís construyen para encerrar a los palestinos, los millones de muros para proteger las propiedades privadas an América Latina de la violencia de la calle. La seguridad privada es quizá el negocio más lucrativo en América Latina hoy. No es por nada que se habla de una 'paramilitarización' del continente (Stålsett, 2002), los 'muros' de control de frontera entre el mundo rico el mundo pobre y hasta el proyecto de construir 'muros' anti-misiles en el espacio. En su impactante discurso en Davos en enero este año el Presidente Lula también hizo referencia a tales muros: muros que separan los que comen de los hambrientos, los que tienen trabajo de los desempleados, los que tienen una vivienda digna de los que viven en la calle o en las miserables favelas, los que tienen acceso a la educación de los analfabetas 1 .... (Lula da Silva, 2003). Pero esta actividad enérgica para 'asegurarse' paradójicamente conlleva mayor inseguridad siempre y cuando sea un intento de olvidar o eliminar la vulnerabilidad humana compartida. La búsqueda de seguridad legítima correspondiendo al derecho a protección en cambio, no se da como un intento de reducir o remover la vulnerabilidad expresada como interdependencia fundamental, sino que busca precisamente condiciones para que el ser humano pueda vivir y desarrollarse como ser vulnerable, abierto a los otros. Esta vida en fragilidad y plenitud es lo que puede expresarse con el segundo concepto ético fundamental frente a la globalización: la dignidad.

\section{Dignidad humana: Requerimiento mínimo y criterio de juicio de un proceso de desarrollo}

En su importante libro Development as Freedom Amartya (1999-2001) argumenta que 
libertad debe ser vista como el objetivo primario tanto como el principal medio del desarrollo. Haciendo una re-definición del concepto de pobreza ('poverty as capability deprivation') Sen (1999: 87-110) nos recuerda un aspecto básico que fue importante para Adam Smith en su obra clásica Wealth of Nations, pero que ha sido olvidado por muchos de sus seguidores autonombrados en la actualidad. Es el significado del auto-estima y auto-respeto de los actores económicos, o mejor dicho, de los pobres (Sen, 1999: 71-88136). Quiero relacionar esto con la importancia ética del concepto de dignidad humana frente a la globalización.

Pobreza obviamente tiene mucho que ver con privación de recursos económicos básicos. Sen subraya que lo importante no es la privación de los recursos en si, sino la privación de capacidades para hacer realidad metas - en otras palabras-, realizar su libertad personal en el desarrollo de sus capacidades y deseos. Dentro de esto, quizás lo más determinante es la privación del sentido de valor personal, el honor, el respeto público. Porque allí radica el fundamento interno y personal para hacer uso de las capacidades presentes (o para poder aguantar y sobrevivir a pesar de la ausencia de estas capacidades). Adam Smith hablaba de la importancia primordial de poder 'appear in public without shame', presentarse públicamente sin vergüenza. Sen lo usa para mostrar que pobreza no se puede medir simplemente con cifras o estándares mínimas, aplicables universalmente. Hasta cierto punto, la pobreza es un fenómeno también interpersonal y social. Este aspecto me parece sumamente importante al tratar el tema de desarrollo y ética, también en la obra clásica de John Rawls (1971:440-446) A Theory of Justice, el autor discute las bases sociales del auto-respeto, y como instituciones y políticas pueden influenciar estas (Citado por Sen, 1999: 327).

Esta arista de la pobreza - la privación del respeto social o público, pobreza como sentido de vergüenza- es relativo, personal y contextual. No se puede medir de manera precisa y generalizable. Pero por eso no es menos importante, porque corresponde a un fenómeno humano reconocido universalmente que se expresa con el término 'dignidad'. La dignidad es íntimamente ligada a la vulnerabilidad humana. Podríamos decir que la dignidad es la fuerza intrínseca de la vulnerabilidad, es su carácter inviolable.

Esta dignidad humana es, en mi opinión, tanto el presupuesto para cualquier proceso de desarrollo, como la meta del mismo. La dignidad es, en otras palabras, requerimiento mínimo y criterio de juicio de un proceso de desarrollo. El gran profeta y líder espiritual Mons. Romero de El Salvador decía que la vida es lo mínimo del máximo don de Dios. Algo parecido se puede decir sobre la dignidad en el proceso de desarrollo. Es un mínimo y un máximo; es un requerimiento y es la meta. Puede haber crecimiento económico, pero si 
en este proceso no se respeta, ni se fortalece la dignidad de las personas humanas, no representa un desarrollo verdadero. La buena sociedad en el sentido ético es una sociedad en la cual todos los seres humanos integrantes de esta sociedad reconocen, respetan y realizan mutuamente su dignidad humana. Este aporte, aunque parecido al aporte de Amartya Sen en el mencionado libro, se distingue de aquel al dar prioridad conceptual a la dignidad en lugar de la libertad. No creo que sean alternativas excluyentes. Al contrario, dignidad y libertad son profundamente relacionadas; se presuponen y se potencian mutuamente. No obstante, el enfoque de la libertad con todos sus méritos excelentemente elaborados por Sen, también tiene algunas limitaciones, lo cual ha mostrado claramente el fracasado modelo neo-liberal. Señalo algunas de ellas en forma de preguntas: 1) ¿Es la libertad realmente una meta, un fin en ś, o más bien, un medio, una capacidad para realizar algo? 2) La capacidad de evitar hambre, muerte prematura por enfermedades curables, así como acceso a educación o al trabajo 6 son adecuadamente definidas como libertades en sí? ¿No son acaso más bien definidas como condiciones para hacer uso de su libertad? 3) ¿Soy realmente libre frente a la vulnerabilidad-dignidad del Otro? Esto lleva a una discusión profunda del carácter 'esencial' de la libertad, en el cual sería fructífero en mi opinión nutrirse del pensamiento sobre libertad que presentan autores tan diversas como p.ej. Martín Lutero, Emmanuel Lévinas y Enrique Dussel. En breve, se podría decir que la libertad del yo en cierto sentido se restringe de la presencia del Otro, pero a la vez que libertad verdadera es ser ligado a ese Otro y poder responder a su llamamiento, poder servirle. En relación con la tesis de Sen, me parece necesario investigar más a fondo qué consecuencias esta limitación de la libertad propia por la defensa o promoción de la libertad de la otra persona tendría para el proceso de desarrollo.

Dignidad consiste de factores externos y factores internos. Sus factores externos son el reconocimiento, el respeto, y las condiciones concretas culturales, materiales, económicas, políticas, entre otras. Para tomar un ejemplo cercano: Asegurar las condiciones para que todos y todas aquí en Brasil puedan comer sus tres comidas al día es un asunto de cubrir necesidades básicas humanas. Pero más que todo, se trata de respetar y conservar la dignidad humana. Así mismo con el acceso a un empleo sustentable $\underline{2}$ o a un pedazo de tierra que se pueda cultivar. La lucha política contra el desempleo y a favor de una reforma agraria que dé una distribución más justa de la tierra es más que todo parte de lo que podríamos Ilamar una 'política de dignidad'.

Igual importancia, sin embargo, tienen los factores internos de la dignidad: Dignidad humana surge del auto-respeto, del auto-estima, de la autoafirmación de la persona. Se trata, en otras palabras, de la necesidad de amarse a si mismo. Pero no como egoísmo. Estamos, como Amartya Sen, lejos de afirmar el mal uso de la sentencia famosa de Smith 
1910 acerca del carnicero, el cervecero y el panadero para promover el egoísmo como la virtud económica por excelencia "No es de la benevolencia del carnicero, del cervecero o del panadero que esperamos nuestra comida, sino de la consideración que ellos hacen de sus propios intereses. Apelamos no a su sentido humanitario sino a su amor por ellos mismos..." (Adam Smith, citado por Sen, 2001). El amor a sí mismo lo entendemos como en el mandamiento báblico "Ama a tu prójimo como a ti mismo" (Levítico 19:18, San Mateo 19:19, par., - análogamente presente en casi todas las grandes religiones). Aquí encontramos una reflejidad, una relacionalidad, una interdependencia profunda, expresada en la pequeña palabra 'como' (como a ti mismo) que conforma el núcleo de la dignidad humana. La vida buena, la vida en plenitud entendida éticamente, depende de esta inter-relacionalidad entre lo interno (amarse a si mismo) y lo externo (amar a su prójimo). Esta interdependencia se puede expresar de varias maneras. En Africa del Sur es común hacer referencias al concepto de ubuntu, que subraya el carácter comunitario de la identidad de cada individuo: 'Yo soy, en cuanto tú eres'. En la misma lógica, también ligado a la interpretación que E. Lévinas hace de la prohibición al asesinato 'No matarás a tu prójimo porque tu lo eres' ha desarrollado la tesis 'asesinato es suicidio' (Hinkelammert, 1996).

Cuando digo que estos factores internos de la dignidad son tan importantes como los factores externos, no quiero de-politizar el concepto. Lo que quiero subrayar es que la dignidad humana puede estar presente a pesar de la falta de un reconocimiento explícito externo de aquella; esto es lo que le da su enorme importancia como fuerza de supervivencia y resistencia en situaciones de marginación y opresión. La fuerza de hacerle frente a dificultades, de resistir, recuperarse, y seguir adelante (lo que en inglés se expresa con las palabras 'coping' y 'resilience') es al mismo tiempo fundamento y efecto de la vulnerabilidad-dignidad humana. Es más originario y va más allá de lo político, sin que en ningún momento se le reste importancia a lo político. Al contrario, la afirmación de dignidad propia lleva a demandas políticas fundamentales. ¿Será por eso que por ejemplo el movimiento zapatista ha tenido un impacto tan grande $y$ ha adherido tantos simpatizantes mundialmente que se trata de una manifestación política de la dignidad de los excluidos? $\underline{3}$.

¿Será por eso que los 'sin tierra' hoy se encuentran entre los actores sociales más importantes en Brasil - con repercusiones significativas en toda la región? ¿Será por eso que se hace cada vez más urgente y prioritario la tarea de promover el papel protagonista de la mujer en los procesos comunitarios de desarrollo? No es por nada que las luchas de las mujeres latinoamericanas, desde las madres y abuelas en Argentina y las viudas en Guatemala, hasta las líderes comunitarias en tantos países..., son reconocidas 
mundialmente como admirables ejemplos de cómo hacer de su vulnerabilidad y dignidad una fuerza de resistencia y nueva vida.

En resumen, la capacidad humana de verse aún en circunstancias contrarias como persona completa, integral y con valor intrínseco es un valor fundamental en el mundo actual de globalización. Por lo tanto el fortalecimiento de esta capacidad, expresado como dignidad humana, debe ser visto como requerimiento mínimo y criterio de juicio ético del proceso de desarrollo.

\section{Justicia: Demanda prioritaria y horizonte último del desarrollo en un mundo globalizado}

Vulnerabilidad, dignidad y justicia: Al proponer que la vulnerabilidad sea reconocida como un valor ético por ser factor antropológico y ético constituyente; y al decir que la dignidad humana últimamente no depende de la actitud o acción política del estado, de los partidos, ni de la sociedad civil o las compañías privadas; es importante al mismo tiempo mantener con fuerza que esto no reduce el significado del tercer valor ético fundamental, la justicia. Hemos definido globalización como conciencia de vulnerabilidad compartida y asimétrica. Aunque en cierto sentido esta asimetría relacional siempre se mantendrá por tratarse de múltiples relaciones unilaterales e incompatibles, subraya que la demanda ética que surge de la interdependencia siempre es unilateral (Løgstrup, 1989), el carácter sistemático y acumulado de esta asimetría crea conciencia de la ausencia de justicia en el mundo globalizado, y se convierten víctimas (en la demanda de aquella. De igual manera, "la irrupción de la dignidad de las víctimas" (Gutiérrez, 1982), a pesar de y en contra de las injusticias es a la vez una expresión de protesta y demanda de justicia. Para Rawls citado por Sen (1999:136) el auto-respeto es quizás el bien más importante de todos al elaborar una teoría de justicia.

¿Qué significa la justicia en un mundo globalizado? ¿Qué forma tomará la justicia? Como en el caso de la libertad o de la equidad, el concepto justicia puede entenderse de varias maneras, entre si divergentes y hasta incompatibles. Volvamos ahora brevemente a mi distinción inicial de dos dimensiones de lo ético-ética como buen comportamiento, buen proceso, o ética como buena meta o buen resultado. Algo parecido podemos decir sobre la justicia. Justicia puede ser simplemente que se cumplen las leyes, que se siguen las reglas del juego. Esto en sí -repito- en América Latina y en el mundo, es sumamente importante. Y a la vez, hay que ir más allá. Inspirado por el concepto teológico de 'justificación', creo que se debe ver la justicia también como inclusión radical frente a leyes jurídicas y económicas que excluyen y deshumanizan. Esta interpretación y aplicación del concepto 
teológico de 'justificación' en un contexto de globalización se inspira en las contribuciones originales y renovadoras de Tamez (1991-1993), y Hinkelammert (1999).

La ley es necesaria. La corrupción y la impunidad frente a la ley tienen efectos desastrosos en la sociedad. La comunidad se construye por medio de leyes cuando estas sean consideradas justas, y por lo tanto son respetadas. Hasta cierto punto, la aplicación de la justicia en el mundo se expresa en las leyes y su cumplimiento. No obstante es una verdad cristiana y humana muy profunda que la ley debe servir a la vida humana y no al revés. Porque cada ley puede llegar al momento en el cual se convierte en una ley que mata, a pesar de que su intención sea buena o su fundamento sea legítimo. Este es el momento en el cual la obediencia de la ley se impone por encima de las necesidades básicas de la vida humana el derecho a la vida digna de todas las personas de la comunidad.

Esta reflexión se inspira en la crítica de la vigencia soteriológica y ética de la ley que encontramos en los evangelios y en las cartas de Pablo. El sábado fue instituido para los seres humanos, no al revés, dice Jesús (San Marco 2: 27, par.) La ley mata, dice Pablo (Romanos 3; Gálatas 3; 1 Corintios 15: 56). 'Justificación’ quiere decir restitución de la vida, vida en dignidad y en plenitud, en contra de la ley que mata.

Cuando en el mundo globalizado se imponen leyes políticas, jurídicas y económicas que llevan a la exclusión creciente de seres humanos, este es una señal de que la ley no sirve a su propósito principal, el propósito de servir a la vida humana, de proteger la persona vulnerable como un ser frágil y digno. Las leyes más peligrosas son las que se establecen como leyes 'naturales', por lo tanto, leyes eternas e incambiables. El cálculo medio-fin, la ley de la eficiencia y la rentabilidad, son leyes consideradas quasi-naturales y eternas en el sistema de globalización. Urge subordinar estas leyes a la lógica de la vida humana.

Aquí encontramos en mi opinión el carácter inmoral del neoliberalismo que ha reinado en América Latina y en casi todo el mundo durante más de una década. Este sistema da prioridad absoluta a la propiedad y al contrato aún al costo de tantas vidas humanas (Myhrvold-Hanssen, 2003). Ahora notamos con satisfacción que cada vez más gente, también líderes políticos, hombres de negocio y economistas, ven que así no se puede seguir. Un sistema que da prioridad a 'leyes' económicas sobre la vida humana no es económicamente sustentable, ni éticamente legítimo. Pero todavía falta mucho para que esta realización al nivel de conciencia y teoría se convierta en realidades políticas.

Es también en esta perspectiva que tenemos que ver la necesidad ética de cancelar las 
deudas impagables e injustas de los países pobres del planeta. La obligación de los pueblos pobres y sufridos de pagar deudas sobre las cuales no han tenido influencia política y de las cuales no han recibido beneficios, tiene que ceder frente a la obligación de dar de comer, de dar salud y educación a nuevas generaciones en estos países. La deuda externa no puede ni debe hacerse eterna.

Es que la justicia va más allá del simple cumplimiento de las reglas y los contratos. La justicia sirve la vida. Por lo tanto tiene aspectos materiales, no sólo formales: Para la gente que vive al margen del sistema, aquellos que no reciben ninguna de las supuestas bendiciones de la globalización, la justicia quiere decir techo, tierra, trabajo, alimentación, educación, salud, entre otras.

Otra vez, estamos ante un mínimo que es un máximo, ante un fundamento que también es horizonte último. Es verdad que la justicia no puede esperar, no se puede posponer. $Y$ a la vez, es verdad que vivimos en un mundo en el cual la justicia completa, la vida en plenitud y dignidad para todos y todas, sigue siendo una esperanza más que una realidad presente. Tenemos que vivir en esta tensión, siempre buscando los pasos primeros, los actos que -aunque parezcan insignificativos- se pueden dar ahora con el fin de un da llegar a la meta. En este sentido las dos dimensiones de la ética con que empecé este ensayo no se contradicen; más bien así se ve una interrelación íntima entre ética como proceso y ética como realización de la buena vida y la buena sociedad global.

Para terminar: En mi opinión, vulnerabilidad, dignidad y justicia son valores éticos indispensables en la tarea de construir un mundo más humano: La vulnerabilidad, por ser -misteriosamente, en medio de lo frágil y lo débil- factor antropológico y ético constituyente. La dignidad, por ser la fuerza que surge de la vulnerabilidad, y que desafía cualquier sistema político, económico y social que no respete, proteja y promueva la persona humana tal como es. La justicia, que más allá del necesario respeto de contratos y leyes requiere una inclusión radical y una defensa incansable de la vida humana, vida en plenitud, vida para todos y todas.

\section{Notas}

1. Véase: World Economic Forum Annual Meeting 2003: Discurso do Presidente Luiz Ignacio Lula da Silva 26 de Janeiro.

2. Véase La importante observación referente al aumento del desempleo como tema ético: “El Premio Nobel de economía Robert Solow critica acerbamente el razonamiento economicista al respecto. Dice Solow que los economistas 
convencionales suponen que si el desempleo es alto, los desocupados buscaran cada vez mas activamente trabajo, y bajaran sus aspiraciones salariales, y ello producirá un equilibrio entre oferta y demanda. La realidad es distinta. Las investigaciones muestran que cuando el desempleo se prolonga la persona se deteriora psicológicamente, su autoestima sufre, su familia es muy afectada, y en lugar de buscar trabajo tiende a retirarse del mercado de trabajo porque no puede tolerar nuevos rechazos. Incluso, se retrae socialmente, porque tiene "vergüenza". Este proceso es muy visible actualmente en América Latina en las clases medias en descenso. Están abandonando sus pertenencias sociales, porque se sienten inferiorizados. Estos sufrimientos humanos son otro tema ético" (Kliksberg, 2001).

3. El Premio Nobel de Literatura José Saramago expresó después de un viaje a Chiapas que los zapatistas habían dado al mundo 'una lección en dignidad'.

\section{Referencias Bibliográficas}

1. Bauman, Zygmunt (1998), Globalization. The Human Consequences. Cambridge: Polity Press.

2. Dussel, Enrique (1992), 1492 El encubrimiento del otro. Hacia el origen del mito de la modernidad. Madrid.

3. Dussel, Enrique (1998), Ética de la Liberación en la Edad de la Globalización y de Ia Exclusión. Madrid Mexico: Editorial Trotta, UAM-I, UNAM.

4. En verden av muligheter - globaliseringens tidsalder og dens utfordringer (2003), Oslo: Det kongelige Utenriksdepartement.

5. Gutiérrez, Germán (1998), Ética y economía en Adam Smith y Friedrich Hayek. San José: DEI.

6. Gutiérrez, Gustavo (1982), La fuerza histórica de los pobres. Salamanca: Ediciones Sígueme.

7. Held, David, David Goldblatt y Jonathan Perraton Anthony McGrew (2000), Global Transformations. Politics, Economics and Culture. Cambridge: Polity Press.

8. Held, David, and Anthony McGrew, eds. (2000), The Global Transformations Reader. An Introduction to the Globalization Debate. Cambridge: Polity Press.

9. Hinkelammert, Franz (1996), “Una sociedad en la que todos quepan: de la impotencia 
de la omnipotencia". In Por una sociedad donde quepan todos, edited by J. Duque. San José: Departamento Ecum énico de Investigaciones.

10. Hinkelammert, Franz J., ed. (1999), El Huracán de la Globalización. San José: Departamento Ecum énico de Investigaciones (DEI).

11. Kliksberg, Bernardo (2003a), Ética y desarrollo; una relación marginada [Internet]. Reproducido de La Gaceta de Económicas, UBA, Buenos Aires, 24 setiembre 2000. Documento incluido dentro de la Biblioteca Digital de la Iniciativa Interamericana de Capital Social, Etica y Desarrollo 2001 [cited 27 June 2003]. Available from www.iadb. org/etica.

12. Kliksberg, Bernardo (2003b), Muchos perdedores, pocos ganadores [Internet]. Reproducido de La Gaceta de Económicas, UBA, Buenos Aires, 24 setiembre 2000. Documento incluido dentro de la Biblioteca Digital de la Iniciativa Interamericana de Capital Social, Etica y Desarrollo 2000 [cited 27 J une 2003]. Available from www.iadb.org/ etica.

13. Lévinas, Emmanuel (1972), Humanisme de I'autre homme. Paris: Fata Morgana.

14. Lula da Silva, Luiz Inacio (2003), Discurso. Paper read at World Economic Forum, 26 Jan, at Davos.

15. Løgstrup, Knud E. (1989), Den etiske fordring. 12. opplag ed. København: Gyldendal.

16. Myhrvold-Hanssen, Thomas L. (2003), Hunger, private property rights, and the right to food [Internet]. University of Oslo, Centre for Development and the Environment, Working Paper 02-2002 2002 [cited June 2003]. Available from http://www.sum.uio.no/publications/ publica.html\#wp.

17. Sáenz, Mario, ed. (2002), Latin American Perspectives on Globalization. Ethics, Politics, and Alternative Visions. Lanham, Boulder, New York, Oxford: Rowman \& Littlefield Publishers, Inc.

18. Sen, Amarty (2003), ¿Qué impacto puede tener la ética? [Internet]. Documento incluido dentro de la Biblioteca Digital de la Iniciativa Interamericana de Capital Social, Etica y Desarrollo. 2001 [cited J une 2003]. Available from www.iadb.org/etica. 
19. Sen, Amarty (2003), What Difference Can Ethics Make? [Internet]. Digital Library of the Inter-American Initiative on Social Capital, Ethics and Development 2001 [cited June 2003]. Available from www.iadb.org./etica/ingles/index-i.htm.

20. Sen, Amartya (1999), Development as Freedom. Oxford - New York: Oxford University Press.

21. Singer, Peter (2002), One World. The Ethics of Globalization. New Haven \& London: Yale University Press.

22. Stålsett, Sturla J. (2002), Sårbarhet og sikkerhet - sett fra Latin-Amerika, 2002. Pacem 5 (2): 147-158.

23. Tamez, Elsa (1991), Contra toda condena: la justificación por la fe desde los excluidos. San José: Editorial Departamento Ecuménico de Investigaciones.

24. Tamez, Elsa (1993), "Justificación". In Conceptos fundamentales del cristianismo, edited by C. Floristan and J.-J. Tamayo. Madrid: Editorial Trotta.

25. http://www. medioambiente.gov. ar/acuerdos/convenciones/unfccc/ccprokio.htm. Consulta:

26. http://sedac. ciesin.org/pidb/texts/rio. declaration. 1992.html. Consulta:

27. http://sedac. ciesin. org/pidb/texts/rio. declaration. 1992. html. Consulta: 